\title{
Effects of Plyometric and Explosive Speed Training on Recreational Marathoners
}

\author{
Christopher J. Lundstrom ${ }^{1}$, Morgan R. Betker ${ }^{1}$ and Stacy J. Ingraham ${ }^{2}$ \\ 1. School of Kinesiology, University of Minnesota-Twin Cities, Minneapolis, MN 55455, USA \\ 2. Mathematics and Science Department, Crown College, St. Bonifacius, MN 55375, USA
}

\begin{abstract}
BACKGROUND: Plyometric training has been shown to improve performance in distance running events up to 5-km, but little research has been done on this type of training for marathon $(42.195-\mathrm{km})$ runners. The purpose of this study was to examine the effects of plyometric and explosive speed training (PLYO) on recreational marathoners. METHODS: Twenty-two subjects (ages 18-23), were randomized to either PLYO or core training (CORE) for a weekly session performed for 12 weeks, in addition to marathon training. Sprint, jump, and distance running performance variables were measured pre- and post-intervention. RESULTS: In the sprint tests, the PLYO group improved in both the $200-\mathrm{m}$ run $(P \leq 0.001)$ and $60-\mathrm{m}$ run $(P=0.004)$, and trended toward improvement in the 30 -m fly $(P=0.051)$. The difference from CORE was significant only in the $200-\mathrm{m}(P=0.002)$. The CORE group did not change in any of the sprint or jump variables. The PLYO group was significantly different from the CORE group in the standing long jump $(P=$ $0.024)$. There were no differences between groups in distance running performance. Both improved in 2-mile $(3.219-\mathrm{km})$ time trial $(\mathrm{p} \leq$ $0.001), \mathrm{VO}_{2 \mathrm{MAX}}(P=0.026$ for CORE; $P=0.002$ for PLYO), and running economy $(P=0.01)$. CONCLUSION: Weekly PLYO training improves sprint speed and maintains jumping ability in recreational marathoners, but does not augment improvements in distance running performance.
\end{abstract}

Key words: Explosive training, jumps training, endurance performance, concurrent training, core training.

\section{Introduction}

Plyometric training consists of high velocity movements performed in rapid succession, with a lengthening (or eccentric) muscle contraction followed quickly by a shortening (concentric) contraction [1]. The most common application of plyometrics is jump training, which consists of sets of jumps, skips, bounding and hops performed with maximal effort and high velocity. Plyometric training is commonly utilized by athletes in sports where jumping and explosive lower body movements are important. Short-duration studies suggest that plyometric training can enhance muscular and neuromuscular function, thereby improving lower limb strength, power and speed [2]. Sprint training (or explosive speed training), which has been shown to increase sprint speed, consists of

Corresponding author: Christopher J Lundstrom, Ph.D., teaching specialist, research field: distance running performance. maximal velocity running efforts of 15 seconds or less with full recovery between repetitions [3].

Explosive speed and plyometric training may have benefits for distance runners. Plyometric training has been shown to improve running economy (RE) and performance in races up to $5-\mathrm{km}$ in length, as well as performance on a number of lower limb power tests in competitive runners [4-10]. Recreational runners may improve RE with a relatively low intensity and volume plyometric program, with no changes in $\mathrm{VO}_{2 \mathrm{MAX}}$ or jump height and efficiency [11]. Explosive speed training improves muscle conduction velocity, an indicator of neuromuscular function linked closely with sprint speed [12]. Both heavy and explosive strength training can improve performance and RE in distance runners [13].

The effects of plyometric and explosive speed training on recreational runners training for a marathon are not known. This population is unique, in that highly 
demanding aerobic training is required of non-competitive, non-elite, and often relatively inexperienced runners. Marathon training may have a detrimental effect on muscle function, strength, and power [14]. However, with effective training and a proper taper, muscle strength and power can be maintained or even improved [15]. Habitual exposure to endurance training at heavy loads has been postulated to lead to chronic, irreversible muscle damage in some athletes [16, 17]. Higher intensity forms of training may be effective in maintaining or improving muscle function $[1,4,5,7,10]$, which has implications related not only to performance, but also to long-term health [18].

Core training, which is widely practiced by runners and other athletes, does not appear to improve running performance or RE [19]. It is often utilized for purported injury prevention benefits by athletes, and while the evidence for this is minimal, a core training group provides a useful, ecologically-valid control group for a randomized-controlled trial. In this study, the use of a core group as a control allowed for matching of training time; both groups did muscular strengthening exercises, but only the plyometric group utilized high velocity and eccentric-overload exercises.

A wide range of physiological, anthropomorphic, and training factors have been identified as related to marathon finish time [20, 21]. The effects of high intensity forms of training on a recreational population is germane given the recent trend among these types of athletes toward participation in intense forms of training. Thus, the purposes of this study were: (1) to determine the effects of plyometric and explosive speed training on sprint speed and jumping ability in college-aged recreational runners engaged in a marathon training program, and (2) to determine whether the effects would transfer to distance running performance.

\section{Materials and Methods}

\subsection{Study Design}

The study was a 12 week exercise intervention utilizing a randomized-controlled parallel group design. One group did plyometric and explosive speed (PLYO) training, and the other did core stability (CORE) training. The outcomes assessed were sprint and jump performance-including vertical jump (VJ), 60-m run (60M), 200-m run (200M), flying 30-m run (30FLY), 10-bound test (10BD), and standing long jump (SLJ) — and distance running performance, including 2-mile (3.219-km) time trial (2MI), a standard 42.195-km marathon (MARA), $\mathrm{VO}_{2 \mathrm{MAX}}$, body mass (BM), running economy (RE) and respiratory exchange ratio (RER). Pre-testing was done in the week prior to the commencement of the intervention. Post-testing was completed within a week of the final training session. The $\mathrm{VO}_{2 \mathrm{MAX}}$ test was done slightly earlier, both pre- and post-intervention.

\subsection{Participants}

Healthy young adults enrolled in a marathon training course were recruited by a study coordinator via email. Inclusion criteria were: enrollment in a university marathon training course, which required a physical and physician's approval to train for and complete a marathon; willingness to commit to an extra 15-20 minute training session once per week and to be randomized into one of two exercise groups.

Exclusion criteria were any pre-existing conditions, such as a muscle or tendon injury, history of injury, or any other health condition that would indicate against the completion of either the PLYO or CORE protocol. Information sessions were conducted and consent was obtained prior to any testing or training sessions. All procedures and protocols were approved by the Institutional Review Board at the University of Minnesota.

A total of 26 subjects were enrolled and randomized, with block randomization (groups of 4) carried out separately for males and females in order to achieve balanced allocation. Two subjects, one from each group, dropped the course within the first month and were excluded from the study. A 
quasi-intention-to-treat analysis was done, with one subject from each group excluded from the analysis due to missing over half of the sessions for reasons unrelated to training or health. One subject in the PLYO group missed over half of the sessions, but cited knee pain as the reason for not wanting to complete the rest of the training sessions. She was included in the spirit of an intention-to-treat analysis. Subjects in the two groups did not differ in age, training sessions completed, or total mileage for the marathon training period. Descriptive data are shown in Table 1.

\subsection{Procedures}

\subsubsection{Training}

Training sessions consisted of one 15-20 minute workout per week. While many studies on distance runners have utilized 2-3 sessions of plyometric training per week $[4,5,7,8,10]$, relatively infrequent training sessions appear to be effective at least for women [22]. The training protocol was low to moderate in volume, and was consistent with what has been previously utilized for less experienced populations [11,23]. The workouts for the two groups were matched for time to complete the training. For both groups, the additional training was progressive in nature, beginning with a lower number of repetitions and sets, and increasing gradually over the course of the training program.

Training sessions were conducted after a Wednesday class session that included a run of approximately 40 minutes of varying intensity, predetermined by the marathon training program. Upon returning from the run, subjects had 10-15 minutes to recover. Sessions were supervised by research staff. Subjects were encouraged to complete all training sessions and exercises if possible, but were advised to stop if an exercise caused sharp pain, injury or excessive discomfort. Subjects who missed part or all of a training session were asked to report their reason. The primary supervisor of the PLYO group was a jumps coach for a local college track \& field team, and the primary supervisor of the CORE group had several years of experience as a strength and conditioning coach. Research staff provided demonstration, instructions, and assistance with proper form and safe execution of exercises.

Both groups performed 6 exercises per session, with the specific exercises alternating weekly. The PLYO exercises involved maximal effort, high velocity jumping and sprinting, with 1-3 sets of 8-20 reps per exercise in the case of the jumping exercises, and 2-4 reps of the sprinting exercises. Specifics of the PLYO protocol are shown in Table 2. The CORE exercises focused on abdominal, back, hip, and gluteal strengthening, and were performed at slow to moderate velocity. The CORE exercises consisted of 1-3 sets of 10-30 reps per exercise or 30-60 seconds for the isometric exercises, as shown in Table 3. For both groups, all exercises were performed with body weight only, with no external resistance or assistance. Approximately one minute of recovery was given between all sets and exercises, with more recovery time allowed.

The marathon training program performed by both groups consisted of a 5 month progressive build-up of mileage. Included in the 5 month build-up, subjects underwent 6 weeks of training prior to the study, which served as a run-in period and allowed standardization

Table 1 Descriptive data of subjects by group.

\begin{tabular}{lll}
\hline & PLYO & CORE \\
\hline $\mathrm{N}$ & 11 & 11 \\
$\mathrm{Sex}$ & $7 \mathrm{~F}, 4 \mathrm{M}$ & $5 \mathrm{~F}, 6 \mathrm{M}$ \\
Age (years) & $20.8 \pm 1.3$ & $19.8 \pm 1.3$ \\
Running volume $(\mathrm{km})$ & $805 \pm 158$ & $763 \pm 185$ \\
Training sessions & $10.6 \pm 2.0$ & $10.8 \pm 1.5$ \\
\hline
\end{tabular}

Age, volume, and training sessions are reported as means \pm S.D. 
Table 2 PLYO training protocol.

\begin{tabular}{|c|c|c|c|c|c|c|c|c|c|c|c|c|c|}
\hline & SPR & SPR & SPR & SPR & HOR & HOR & HOR & HOR & HOR & VERT & VERT & VERT & VERT \\
\hline Sess & 50 BLD & $30 \mathrm{FLY}$ & 60 SPR & INO & FH & ALB & S.Leg FH & Lat. CJ & F/B CJ & SJ & SSJ & DJ & BJ \\
\hline 1 & 2 & 2 & & & $1 \times 10$ & $2 \times 10$ & $1 \times 8$ & & & $1 \times 10$ & & & \\
\hline 2 & & & 2 & 2 & & & & $1 \times 10$ & $1 \times 10$ & & $1 \times 10$ & $1 \times 8$ & \\
\hline 3 & 3 & 3 & & & $2 \times 8$ & $2 \times 15$ & $1 \times 12$ & & & $2 \times 8$ & & & \\
\hline 4 & & & 3 & 3 & & & & $1 \times 15$ & $1 \times 15$ & & $2 \times 8$ & $1 \times 12$ & \\
\hline 5 & 3 & 3 & & & $2 \times 10$ & $2 \times 20$ & $2 \times 10$ & & & $2 \times 10$ & & & \\
\hline 6 & & & 3 & 3 & & & & $2 \times 10$ & $2 \times 10$ & & $2 \times 10$ & $1 \times 15$ & \\
\hline 7 & 4 & 4 & & & $2 \times 15$ & $3 \times 15$ & $2 \times 12$ & & & $2 \times 15$ & & & \\
\hline 8 & & & 4 & 4 & & & & $2 \times 15$ & $2 \times 15$ & & $2 \times 15$ & & $1 \times 10$ \\
\hline 9 & 4 & 4 & & & $2 \times 15$ & $3 \times 20$ & $2 \times 15$ & & & $2 \times 15$ & & & \\
\hline 10 & & & 4 & 4 & & & & $2 \times 20$ & $2 \times 20$ & & $2 \times 15$ & & $1 \times 15$ \\
\hline 11 & 3 & 3 & & & $1 \times 20$ & $2 \times 20$ & $1 \times 20$ & & & $1 \times 20$ & & & \\
\hline 12 & & & 2 & 2 & & & & $1 \times 20$ & $1 \times 20$ & & $1 \times 20$ & & $1 \times 10$ \\
\hline
\end{tabular}

Sess $=$ session number. SPR $=$ sprint exercise, HOR $=$ horizontal jump exercise, VERT $=$ vertical jump exercise. Exercises abbreviations: $50 \mathrm{BLD}=50-\mathrm{m}$ build up to max. velocity, $30 \mathrm{FLY}=30-\mathrm{m}$ fly with a 20-m acceleration zone, $60 \mathrm{SPR}=60-\mathrm{m}$ sprint at max. velocity, INO = an in-and-out sprint consisting of a 20-m acceleration zone, 10-m max. velocity zone, 20-m float, and a second 10-m max. velocity zone, FH = frog hops, ALB = alternate leg bounds, S.Leg FH = single leg forward hops, Lat. CJ = lateral cone jumps, F/B CJ = forward/backward cone jumps, SJ = squat jumps, SSJ = split scissor jumps (or lunge jumps), DJ = depth jumps, and BJ = box jumps from a self-selected box height. All jumps were performed at maximal intensity and repeatedly, without rest between repetitions.

Table 3 CORE training protocol.

\begin{tabular}{|c|c|c|c|c|c|c|c|c|c|c|c|c|}
\hline & ABS & ABS & ABS & ABS & BACK & BACK & BACK & BACK & HIP/GL & HIP/GL & HIP/GL & HIP/GL \\
\hline Sess. \# & $\mathrm{CR}$ & SCR & SU & VS & SM & $\mathrm{BU}$ & PK & SPK & $\mathrm{FH}$ & SBA & BDG & $\mathrm{BD}$ \\
\hline 1 & $1 \times 20$ & & $1 \times 20$ & & $1 \times 20$ & & $30 \mathrm{~s}$ & & $1 \times 20$ & & $1 \times 20$ & \\
\hline 2 & & $1 \times 20$ & & $1 \times 20$ & & $1 \times 10$ & & $30 \mathrm{~s}$ & & $1 \times 20$ & & $1 \times 20$ \\
\hline 3 & $1 \times 30$ & & $1 \times 30$ & & $1 \times 30$ & & $45 \mathrm{~s}$ & & $1 \times 30$ & & $1 \times 30$ & \\
\hline 4 & & $1 \times 30$ & & $1 \times 30$ & & $1 \times 15$ & & $45 \mathrm{~s}$ & & $1 \times 30$ & & $1 \times 30$ \\
\hline 5 & $2 \times 20$ & & $2 \times 20$ & & $2 \times 20$ & & $60 \mathrm{~s}$ & & $2 \times 20$ & & $2 \times 20$ & \\
\hline 6 & & $2 \times 20$ & & $2 \times 20$ & & $2 \times 10$ & & $60 \mathrm{~s}$ & & $2 \times 20$ & & $2 \times 20$ \\
\hline 7 & $2 \times 30$ & & $2 \times 30$ & & $2 \times 30$ & & $2 \times 45 s$ & & $2 \times 30$ & & $2 \times 30$ & \\
\hline 8 & & $2 \times 30$ & & $2 \times 30$ & & $2 \times 15$ & & $2 \times 45 s$ & & $2 \times 30$ & & $2 \times 30$ \\
\hline 9 & $3 \times 30$ & & $3 \times 30$ & & $3 \times 30$ & & $2 \times 60 s$ & & $3 \times 30$ & & $3 \times 30$ & \\
\hline 10 & & $3 \times 30$ & & $3 \times 30$ & & $3 \times 15$ & & $2 \times 60 s$ & & $3 \times 30$ & & $3 \times 30$ \\
\hline 11 & $2 \times 30$ & & $2 \times 30$ & & $2 \times 30$ & & $60 \mathrm{~s}$ & & $2 \times 30$ & & $2 \times 30$ & \\
\hline 12 & & $2 \times 30$ & & $2 \times 30$ & & $2 \times 15$ & & $60 \mathrm{~s}$ & & $2 \times 30$ & & $2 \times 30$ \\
\hline
\end{tabular}

Sess $=$ session number. ABS $=$ abdominal exercise. $\mathrm{BACK}=$ back exercise. HIP/GL $=$ hip or gluteal exercise. Exercise abbreviations: $\mathrm{CR}=$ crunches, $\mathrm{SCR}=$ side crunches (per side), $\mathrm{SU}=$ sit-ups, $\mathrm{VS}=\mathrm{V}$ sit-ups, $\mathrm{SM}=$ supermans, $\mathrm{BU}=$ back-ups or back extensions, using a training table, $\mathrm{PK}=$ plank, $\mathrm{SPK}=$ side plank (time per side), $\mathrm{FH}=$ fire hydrant, $\mathrm{SBA}=$ Swiss Ball adductors, $\mathrm{BDG}=$ bridging, $\mathrm{BD}=$ bird dog. All exercise were performed at slow to moderate velocity.

of training status. Subjects ran 4-5 days per week, with an average weekly mileage of $35-\mathrm{km}$ per week and a peak of $60-\mathrm{km}$ per week. Long runs were done weekly, and built up to $32-\mathrm{km}$ by the peak training period.

\subsubsection{Anthropometric Characteristics}

Height and weight were measured pre- and post-, prior to the treadmill RE test. Subjects removed footwear, and height was measured using an Accustat Genentech Stadiometer (San Francisco, CA, USA). Weight was measured in pounds to the nearest tenth using a ProDoc Detecto (PD300) scale (Webb City, MO, USA) and converted to kilograms for analysis. 
During weighing, subjects wore light, minimal clothing.

2.3.3. Sub-maximal 30 Minute Running Test and $\mathrm{VO}_{2 \mathrm{MAX}}$ Test

For the sub-maximal running test, subjects ran on a motorized Woodway Pro XL 27 treadmill (Waukesha, WI, USA) for a total of 33 continuous minutes, including a 3 minute warm-up and 6 stages of 5 minutes each. They were advised to follow a dietary regiment similar to that which they typically use prior to a race or challenging workout. Stages were calculated off recent $2 \mathrm{MI}$ finish time. The warm-up was done at $63 \%$ of $2 \mathrm{MI}$ velocity, and the 6 stages of 5 minutes each were at $68 \%, 73 \%, 78 \%, 83 \%, 88 \%$, and $93 \%$ of 2 -mile velocity. The final stage velocity is equivalent to a predicted $10 \mathrm{k}$ velocity, based on previously published race pace conversion charts [24]. Though this was not meant to be a maximal effort, the duration and intensity were moderately challenging. Research staff emphasized that if at any point the subject wished to stop the test, he or she was free to do so. A face mask and pneumatech were worn for gas analysis via Medgraphics Ultima CPX metabolic cart (MCG Diagnostics, St. Paul, MN, USA). Standard calibration procedures were used prior to each testing session. In order to assess metabolic response to exercise of moderate duration over a range of sub-maximal paces, RER and RE in $\mathrm{ml} / \mathrm{kg} / \mathrm{km}$ were calculated by taking average values over the 30 minutes. Stage-by-stage analysis was considered, but shed no additional light on the variables of interest.

The $\mathrm{VO}_{2 \mathrm{MAx}}$ test was performed with the same treadmill and metabolic cart on a separate visit. The same procedures were used for height and weight. A graduated protocol was utilized, with velocity increasing each minute up to 2 -mile velocity at $1.0 \%$ incline, and then increasing in incline by $1.5 \%$ each minute until volitional exhaustion was reached.

\subsubsection{Running Performance Assessment}

The 2MI time trials were conducted as part of the university course curriculum, as was the MARA. The
2MI runs were held on a non-banked, Mondo (Conshohocken, PA, USA) 200-m indoor track. Each runner had a lap counter who marked the completion of each lap and recorded 1-mile split time and 2MI finish time on a data collection sheet. The pre-test 2MI was completed at the start of the marathon training class, one week prior to the initiation of the training intervention. The post-test was completed near the conclusion of the training intervention, 10 days prior to the MARA. The MARA was run on a certified course as part of an event open to the general public. Chip times were gathered from online race results, and checked against training logs for accuracy. Predicted marathon time was calculated from the post-test 2MI using race time predictor charts [24]. Difference between actual and predicted MARA time is reported as a percentage.

\subsubsection{Sprint and Jump Testing}

Sprint and jump tests were conducted on the same indoor track as the 2MI, and consisted of the following, performed in this order: $30 \mathrm{FLY}, \mathrm{SLJ}, 60 \mathrm{M}$, and 10BD and 200M. A standard warm-up (5 minute jog, and 10 minutes of dynamic warm-up drills and accelerations) was performed prior to the commencement of testing. The sprint tests were timed electronically using Brower Timing Systems TC-Timing wireless timing gates (Draper, UT, USA) set at waist height. The 30FLY, with a $20-\mathrm{m}$ acceleration zone, was timed using two sets of timing gates. The $60 \mathrm{M}$ and $200 \mathrm{M}$ were timed using the touchpad starter, set to start on release, and one set of timing gates. The 10BD consisted of a rock-step start, into 10 continuous alternate leg strides down the long jump runway. Subjects were instructed to cover as much distance as possible. The SLJ was done into a standard sand landing pit at the indoor track facility. Jump measurements were taken using a fiberglass measuring tape (Empire, Mukwonago, WI, USA) with two researchers spotting and measuring. Distances of all trials were recorded immediately by research staff. Distance was measured from the scratch line to furthest back point of contact upon landing (in 
most cases, the heel).

Subjects were given 3 attempts to produce maximal efforts in the jumps, with a minimum of a 1-minute rest between attempts, and at least a 3-minute rest between tests (ICC: SLJ $=0.987,10 \mathrm{BD}=0.989$ ). They were given a single attempt in each of the sprints, to minimize the effects of fatigue during the testing session. The 200M was done last as it was likely produce greater fatigue than the other tests. All tests were explained carefully and demonstrated to subjects prior to testing. All timing and measurement procedures were performed consistently and were the same for pre- and post-testing.

On a separate visit, prior to the submaximal 30-minute running test, VJ was assessed using the Vertec Jump and Reach system (Huntington Beach, CA, USA). Subjects performed a self-paced 5 minute warm-up jog on a treadmill and were given the opportunity to perform practice jumps. Three minutes of rest were taken between the warm-up and the VJ. Calibration of the Vertec was done for each subject. Subjects performed 3-6 maximal effort countermovement jumps with stationary feet, with at least 30 seconds of rest between attempts. Subjects performed at least three attempts, and continued until they failed to improve on two consecutive attempts, with a maximum of 6 jumps allowed.

\subsection{Statistics}

Means and standard deviations were calculated for each measure. Data were tested for normality and homoscedasticity using the Shapiro-Wilk and Levene tests. The two groups of dependent variables, sprint/jump performance and distance running performance, were assessed separately using MANOVA. Baseline measures were analyzed using independent samples t-tests to detect differences between the groups before the intervention. Paired samples t-tests were used to assess changes from baseline by group. Between groups analysis on the dependent variables was calculated based on percent change from pre- to post-testing. Independent samples t-tests or the Mann-Whitney U Test was used to compare the groups. A MANOVA was used to assess differences in the effect of training by sex and performance level. Performance level was determined based on pre-test $2 \mathrm{MI}$ time, with the top 6 males and females being assigned to the FAST group, and the remainder ( 7 females and 5 males) being assigned to the SLOW group. Univariate ANOVA was also used for post-hoc analysis to explore for interactions by performance level and sex. Statistical analysis was done using SPSS (version 21.0; IBM, Armonk, NY, USA).

\section{Results}

Baseline analysis showed no differences between groups, though there was a trend toward lower BM in the PLYO group $(P=0.063)$. Sprint and jump results are presented in Table 4. Distance running performance results are shown in Table 5. Pre- and post-test values and percent change are reported for all variables. Assessment with MANOVA showed a significant effect of training group on the dependent variables related to sprint and jump performance $(P=0.045)$, but not for those related to distance running performance. There was no interaction between training group and either sex or performance level for the sprint/jump or distance running dependent variables. Results are shown for men and women independently, but they were pooled for analysis.

The CORE group did not change from baseline in any of the sprint or jump variables, but had a trend toward a decrement in performance in the SLJ $(P=$ $0.067)$. The PLYO group improved in both the $200 \mathrm{M}$ $(P \leq 0.001)$ and $60 \mathrm{M}(P=0.004)$, and showed a strong trend toward improvement in the 30MFLY $(P=0.051)$. In the distance running performance variables, both groups improved in $2 \mathrm{MI}(P \leq 0.001), \mathrm{VO}_{2 \mathrm{MAX}}(P=$ 0.026 for CORE; $P=0.002$ for PLYO), and RE ( $P=$ $0.01)$. Both groups were also significantly slower $(P \leq$ 0.001 for CORE; $P=0.004$ for PLYO) than predicted in the marathon. 
Table 4 Pre- and post-training sprint and jump performance variables.

\begin{tabular}{|c|c|c|c|c|c|c|c|c|}
\hline \multirow{2}{*}{ Variable } & & \multicolumn{3}{|c|}{$\operatorname{CORE}(n=11,5$ women $)$} & \multicolumn{4}{|c|}{ PLYO ( $n=11,7$ women $)$} \\
\hline & & Pre- & Post- & $\%$ change & Pre- & Post- & $\%$ change & $P=$ \\
\hline \multirow{3}{*}{$200 \mathrm{M}(\mathrm{sec})$} & ALL & $34.28 \pm 5.39$ & $33.78 \pm 4.89$ & $-1.3 \pm 3.0$ & $36.52 \pm 6.24$ & $34.64 \pm 6.46 \dagger$ & $-5.8 \pm 3.7 \ddagger$ & 0.006 \\
\hline & M & $29.94 \pm 1.27$ & $29.88 \pm 1.71$ & $-0.3 \pm 3.5$ & $31.64 \pm 4.52$ & $29.20 \pm 4.81$ & $-8.6 \pm 2.5$ & \\
\hline & W & $39.49 \pm 2.92$ & $38.47 \pm 2.41$ & $-2.6 \pm 1.9$ & $39.31 \pm 5.45$ & $37.75 \pm 5.19$ & $-4.1 \pm 3.3$ & \\
\hline \multirow{3}{*}{$60 \mathrm{M}(\mathrm{sec})$} & ALL & $9.83 \pm 1.40$ & $9.67 \pm 1.19$ & $-1.8 \pm 7.2$ & $10.45 \pm 1.37$ & $9.88 \pm 1.51 \dagger$ & $-6.2 \pm 6.9$ & 0.154 \\
\hline & M & $8.74 \pm 0.55$ & $8.82 \pm 0.89$ & $0.4 \pm 9.0$ & $9.54 \pm 1.08$ & $8.59 \pm 0.79$ & $-11.1 \pm 8.6$ & \\
\hline & W & $11.15 \pm 0.74$ & $10.69 \pm 0.44$ & $-4.3 \pm 3.9$ & $10.97 \pm 1.29$ & $10.62 \pm 1.33$ & $-3.4 \pm 4.2$ & \\
\hline \multirow{3}{*}{ 30FLY (sec) } & ALL & $4.23 \pm 0.77$ & $4.20 \pm 0.56$ & $-0.3 \pm 6.1$ & $4.44 \pm 0.72$ & $4.31 \pm 0.67$ & $-3.0 \pm 2.9$ & 0.191 \\
\hline & M & $3.66 \pm 0.20$ & $3.78 \pm 0.30$ & $3.0 \pm 3.0$ & $3.84 \pm 0.38$ & $3.72 \pm 0.30$ & $-3.3 \pm 3.0$ & \\
\hline & W & $4.91 \pm 0.60$ & $4.71 \pm 0.30$ & $-4.2 \pm 7.0$ & $4.78 \pm 0.65$ & $4.64 \pm 0.57$ & $-2.9 \pm 3.4$ & \\
\hline \multirow{3}{*}{ SLJ (m) } & ALL & $2.22 \pm 0.54$ & $2.12 \pm 0.45$ & $-4.5 \pm 7.6$ & $1.98 \pm 0.41$ & $2.02 \pm 0.38$ & $1.9 \pm 4.1 \dagger$ & 0.024 \\
\hline & M & $2.62 \pm 0.34$ & $2.45 \pm 0.28$ & $-6.9 \pm 8.3$ & $2.37 \pm 0.40$ & $2.40 \pm 0.29$ & $1.7 \pm 5.5$ & \\
\hline & W & $1.73 \pm 0.13$ & $1.71 \pm 0.20$ & $-1.6 \pm 6.3$ & $1.76 \pm 0.22$ & $1.80 \pm 0.22$ & $2.0 \pm 3.6$ & \\
\hline \multirow{3}{*}{ 10BD (m) } & ALL & $21.5 \pm 3.2$ & $20.7 \pm 3.3$ & $-4.2 \pm 7.6$ & $19.8 \pm 2.6$ & $19.8 \pm 2.7$ & $-0.5 \pm 6.9$ & 0.332 \\
\hline & $\mathrm{M}$ & $23.8 \pm 2.0$ & $22.8 \pm 3.2$ & $-5.4 \pm 8.4$ & $22.3 \pm 1.8$ & $22.7 \pm 1.2$ & $1.8 \pm 5.6$ & \\
\hline & W & $18.6 \pm 1.5$ & $18.1 \pm 0.6$ & $-2.8 \pm 7.2$ & $18.4 \pm 1.7$ & $18.1 \pm 1.7$ & $-1.9 \pm 7.6$ & \\
\hline \multirow{3}{*}{ VJ (m) } & ALL & $0.576 \pm 0.193$ & $0.554 \pm 0.142$ & $-3.0 \pm 8.2$ & $0.513 \pm 0.127$ & $0.503 \pm 0.114$ & $-1.6 \pm 6.1$ & 0.847 \\
\hline & $\mathrm{M}$ & $0.719 \pm 0.147$ & $0.663 \pm 0.086$ & $-8.0 \pm 13.3$ & $0.645 \pm 0.076$ & $0.617 \pm 0.099$ & $-5.6 \pm 10.9$ & \\
\hline & W & $0.409 \pm 0.038$ & $0.424 \pm 0.058$ & $3.0 \pm 8.2$ & $0.437 \pm 0.074$ & $0.439 \pm 0.064$ & $0.7 \pm 4.6$ & \\
\hline
\end{tabular}

All results are \pm S.D. $P$-values are two-tailed, based on t-tests of $\%$ change by group. ALL $=$ all subjects. $\mathrm{M}=$ men. $\mathrm{W}=$ women. Statistical analysis done for ALL, and M, W values provided for reference. $†$ Significantly different from baseline. $\ddagger$ Significantly different from CORE group $(P \leq 0.05)$.

Table 5 Pre- and post-training distance running performance variables.

\begin{tabular}{|c|c|c|c|c|c|c|c|c|}
\hline \multirow[b]{2}{*}{ Variable } & & \multicolumn{3}{|c|}{ CORE ( $n=11,5$ women $)$} & \multicolumn{4}{|c|}{ PLYO ( $n=11,7$ women $)$} \\
\hline & & Pre- & Post- & $\%$ change & Pre- & Post- & $\%$ change & $P$-value \\
\hline \multirow{3}{*}{$\begin{array}{l}\mathrm{VO}_{2 \mathrm{MAX}} \\
(\mathrm{ml} / \mathrm{kg} / \mathrm{min})\end{array}$} & ALL & $53.6 \pm 8.3$ & $57.4 \pm 7.5 \dagger$ & $6.5 \pm 7.9$ & $50.5 \pm 8.8$ & $57.8 \pm 8.3 \dagger$ & $12.5 \pm 9.7$ & 0.124 \\
\hline & M & $59.1 \pm 6.4$ & $62.2 \pm 5.6$ & $4.7 \pm 10.6$ & $55.2 \pm 13.0$ & $65.4 \pm 4.7$ & $16.2 \pm 14.5$ & \\
\hline & $\mathrm{W}$ & $47.1 \pm 4.7$ & $51.5 \pm 4.9$ & $8.7 \pm 2.3$ & $47.7 \pm 4.3$ & $53.5 \pm 6.6$ & $10.4 \pm 5.4$ & \\
\hline \multirow{3}{*}{$\begin{array}{l}2 \text { mile TT } \\
(\mathrm{min})\end{array}$} & ALL & $14.0 \pm 2.0$ & $13.3 \pm 1.7 \dagger$ & $-5.1 \pm 2.2$ & $14.7 \pm 2.4$ & $14.0 \pm 2.3 \dagger$ & $-4.3 \pm 3.1$ & 0.521 \\
\hline & $\mathrm{M}$ & $12.8 \pm 0.9$ & $12.1 \pm 0.8$ & $-5.0 \pm 2.3$ & $12.5 \pm 2.2$ & $12.2 \pm 2.5$ & $-2.6 \pm 3.1$ & \\
\hline & $\mathrm{W}$ & $15.5 \pm 2.0$ & $14.7 \pm 1.5$ & $-5.3 \pm 2.3$ & $16.0 \pm 1.3$ & $15.1 \pm 1.4$ & $-5.4 \pm 2.8$ & \\
\hline \multirow{3}{*}{$\begin{array}{l}\text { Marathon } \\
(\min )\end{array}$} & ALL & $202.5 \pm 25.0$ & $237.1 \pm 31.6 \dagger$ & $14.5 \pm 4.0$ & $213.9 \pm 33.5$ & $255.5 \pm 66.2 \dagger$ & $14.0 \pm 10.2$ & 0.891 \\
\hline & M & $185.7 \pm 12.1$ & $217.8 \pm 14.2$ & $14.7 \pm 3.9$ & $186.6 \pm 36.8$ & $218.7 \pm 91.2$ & $10.1 \pm 14.8$ & \\
\hline & $\mathrm{W}$ & $222.7 \pm 21.3$ & $260.4 \pm 31.7$ & $14.2 \pm 4.5$ & $229.5 \pm 20.3$ & $276.5 \pm 41.5$ & $16.2 \pm 7.0$ & \\
\hline \multirow{3}{*}{$\begin{array}{l}\text { Body Mass } \\
(\mathrm{kg})\end{array}$} & ALL & $73.5 \pm 9.6$ & $72.9 \pm 9.6$ & $-0.9 \pm 3.0$ & $65.8 \pm 8.5$ & $65.8 \pm 7.8$ & $0.0 \pm 3.5$ & 0.510 \\
\hline & M & $79.8 \pm 8.0$ & $79.9 \pm 6.5$ & $0.2 \pm 3.2$ & $71.8 \pm 4.8$ & $71.5 \pm 1.8$ & $-0.4 \pm 4.4$ & \\
\hline & $\mathrm{W}$ & $65.9 \pm 4.4$ & $64.5 \pm 3.8$ & $-2.2 \pm 2.6$ & $62.4 \pm 8.5$ & $62.5 \pm 8.1$ & $0.2 \pm 1.9$ & \\
\hline \multirow{3}{*}{$\begin{array}{l}\mathrm{RE} \\
(\mathrm{ml} / \mathrm{kg} / \mathrm{km})\end{array}$} & ALL & $218.6 \pm 19.4$ & $192.9 \pm 37.0 \dagger$ & $-16.5 \pm 20.5$ & $216.3 \pm 17.9$ & $200.2 \pm 29.5 \dagger$ & $-9.6 \pm 13.2$ & 0.519 \\
\hline & M & $219.9 \pm 18.7$ & $193.7 \pm 36.0$ & $-16.0 \pm 16.6$ & $211.9 \pm 17.3$ & $198.4 \pm 24.7$ & $-7.3 \pm 6.5$ & \\
\hline & W & $217.1 \pm 22.3$ & $192.0 \pm 42.3$ & $-17.2 \pm 26.5$ & $218.8 \pm 19.0$ & $201.2 \pm 33.7$ & $-10.9 \pm 16.3$ & \\
\hline \multirow{3}{*}{ RER } & ALL & $1.031 \pm 0.048$ & $1.049 \pm 0.096$ & $1.3 \pm 6.3$ & $1.028 \pm 0.048$ & $0.997 \pm 0.045$ & $-3.4 \pm 6.7$ & 0.112 \\
\hline & M & $1.047 \pm 0.054$ & $1.105 \pm 0.087$ & $4.9 \pm 6.1$ & $1.023 \pm 0.041$ & $1.013 \pm 0.038$ & $-1.1 \pm 4.0$ & \\
\hline & W & $1.012 \pm 0.035$ & $0.982 \pm 0.058$ & $-3.1 \pm 2.9$ & $1.031 \pm 0.054$ & $0.988 \pm 0.049$ & $-4.7 \pm 7.9$ & \\
\hline
\end{tabular}

All results are \pm S.D. P-values are two-tailed, based on t-tests of $\%$ change by group. ALL = all subjects. $\mathrm{M}=$ men. $\mathrm{W}=$ women. Statistical analysis done for ALL, and M, W values provided for reference. Marathon: pre = predicted, post = actual finish time. $\dagger$ Significantly different from baseline. 
There were significant differences between PLYO and CORE in the 200M $(P=0.006)$ and the SLJ $(P=$ $0.024)$. The PLYO group improved $5.7 \%$ compared to $1.3 \%$ for CORE in the $200 \mathrm{M}$, and improved $1.9 \%$ vs. a 4.5\% decrement in CORE for the SLJ. The PLYO group improved more than CORE in the other sprint variables, but the differences were not significant. Changes in sprint performance by group are shown in Fig. 1. In all of the jump variables, CORE decreased non-significantly in jump distance or height, whereas PLYO increased non-significantly in SLJ and decreased, but to a lesser degree than CORE, in 10BD and VJ, as shown in Fig. 2.

In the distance running performance variables, there were no significant differences between the groups. In the $2 \mathrm{MI}$, improvements were very similar between the groups, with CORE improving by $5.1 \%$ vs. $4.3 \%$ in PLYO $(P=0.521)$. Marathon time as a function of predicted times was also very similar between the groups, with the PLYO running $14.0 \%$ slower than predicted and CORE running $14.5 \%$ slower $(P=0.891)$. No change was seen in BM in either group. There was no difference in the change in RE between the groups, with CORE improving by $16.5 \%$ and PLYO improving

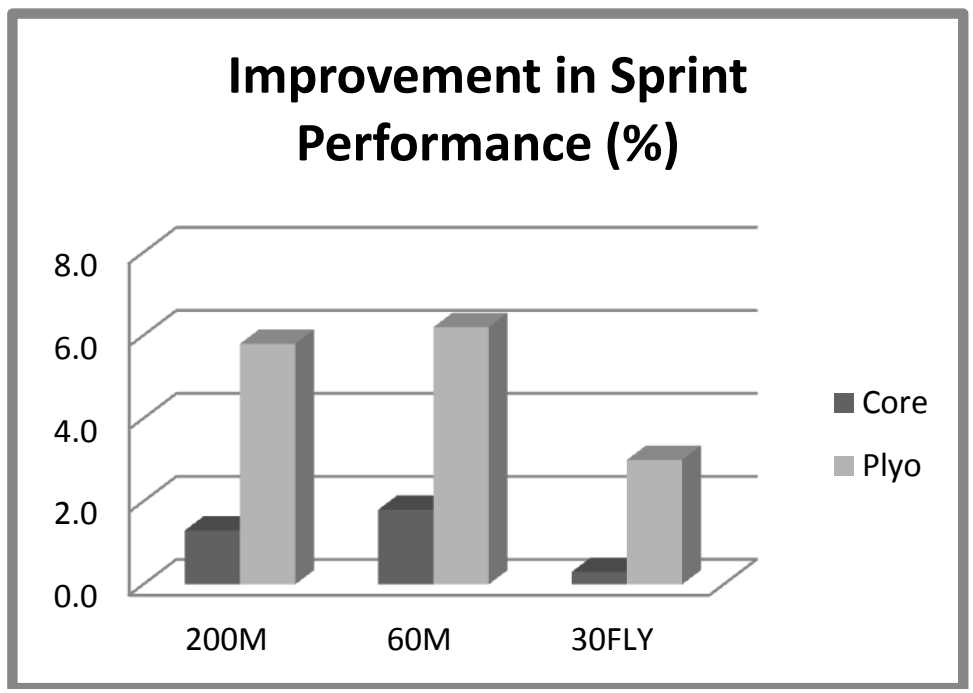

Fig. 1 Percentage improvement in sprint performance by group for the 200M, 60M, and 30MFLY.

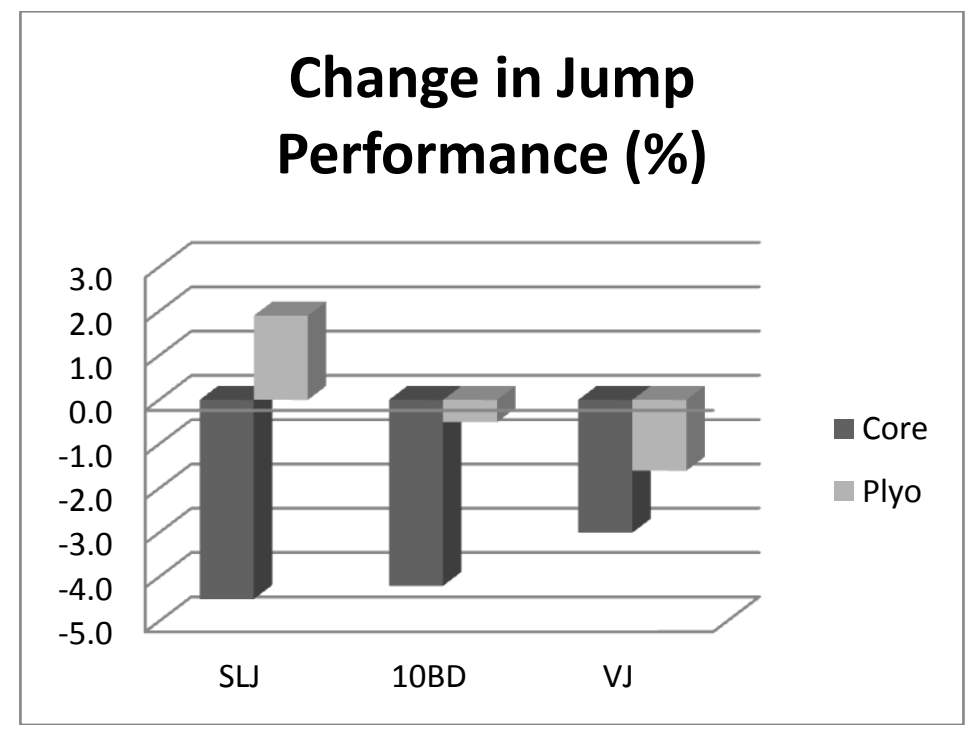

Fig. 2 Percentage change in jump performance by group for the SLJ, 10BD, and VJ. 


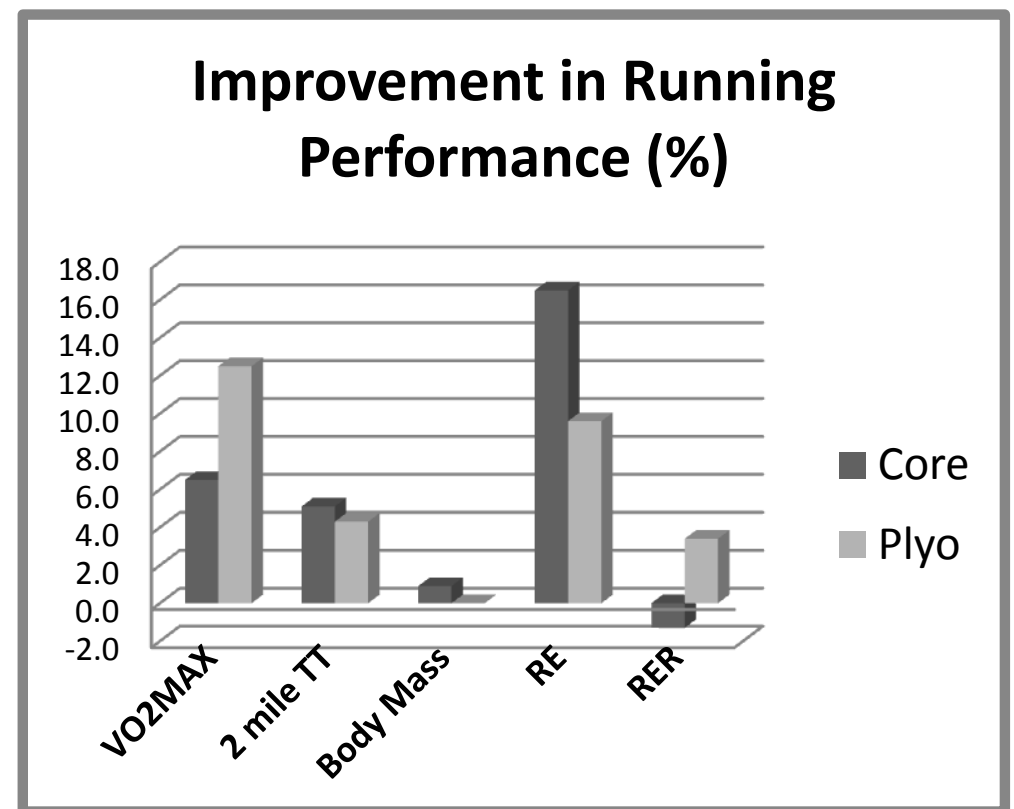

Fig. 3 Percentage improvement by group in distance running performance variables: $\mathrm{VO}_{2 \mathrm{MAx}}$, 2MI, BM, RE, and RER. Reductions in 2MI, BM, RE (per km), and RER are inverted here in a positive direction, so that all positive percentages indicate adaptations consistent with improvement in running performance.

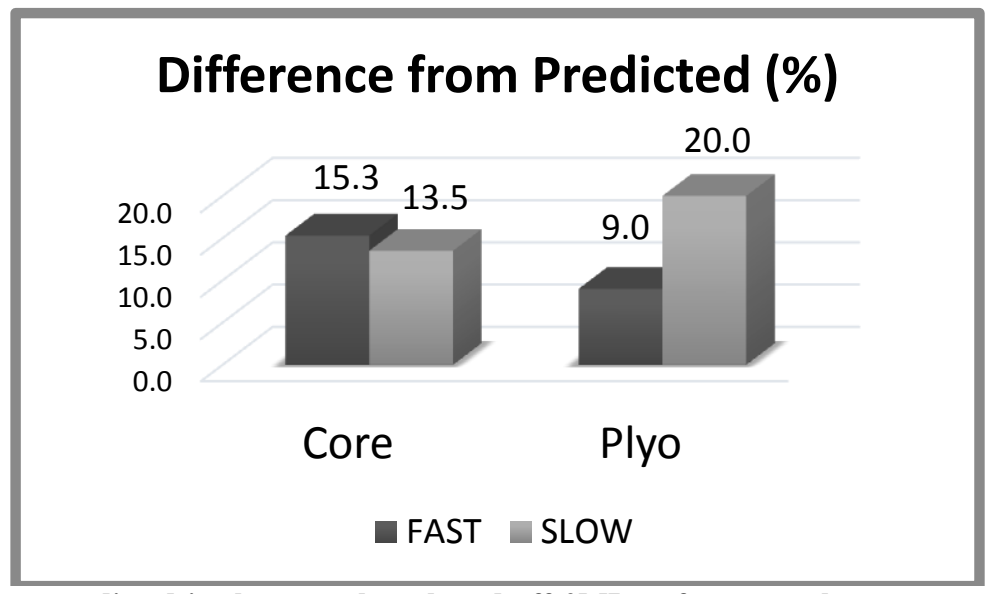

Fig. 4 Percent slower than predicted in the marathon, based off 2MI performance, by group and performance level. The cut-points were 12.5 minutes for men and 15.5 minutes for women.

by $9.6 \%(P=0.519)$. In $\mathrm{VO}_{2 \mathrm{MAX}}$, PLYO improved by $12.5 \%$ vs. $6.5 \%$ in CORE $(P=0.124)$. Average RER dropped by $3.4 \%$ in PLYO, but increased $1.3 \%$ in CORE $(P=0.112)$. A univariate ANOVA of difference from predicted marathon time, including performance level and training group, showed a significant interaction between training group and performance level $(P=0.047)$, with the FAST runners from the PLYO group performing better in the marathon than those in the CORE group, but the SLOW runners in the PLYO group performing worse than those in the CORE group.

\section{Discussion}

This study examined the effects of a 12 week plyometric training program on sprint and jump performance, and distance running performance in college-aged recreational marathon runners. This study was novel in examining this type of training for marathon runners. While marathon training and plyometric training have very different physiological aims, it appears that among this population, beneficial 
adaptations specific to both types of training can be made when training is done concurrently.

Sprint performance improved and jump performance did not decline in the PLYO group, whereas the CORE group did not change in sprint performance, and decreased non-significantly in measures of jump performance. The frequency and volume of PLYO training was relatively low in comparison with other studies of distance runners training for shorter races $[4$, $5,7,8,10,11]$. The lower frequency and volume of training in this program was intentionally conservative, as the primary concern for this population was completion of the marathon, and not to maximize speed and/or power gains. However, it appears that this low volume was effective in improving speed and maintaining jumping performance. Greater improvements may be possible with a more aggressive training protocol in those who are able to tolerate such a program. Intermittent testing and monitoring throughout the training period could also be considered, with volume and frequency adjusted based on individual adaptation and tolerance for training.

The failure of the PLYO training to produce an improvement in jump variables is somewhat surprising given the abundance of literature on PLYO training and improved jump performance [25]. The two dropouts from the study and two exclusions from analysis led to an imbalance between men and women in the groups, with only four men in the PLYO group and six in the CORE group. Given the evidence that women may not have the same magnitude of response to PLYO training, specifically in terms of jump performance [25], this could have reduced the power of the present study to find differences between the groups. However, no interaction between sex and training group was detected. The decrement in jump performance seen in the CORE group is similar to findings in other studies on heavy endurance training $[8,26]$. In those studies, the control group experienced a decrement in jump performance, while the strength training group [26] or plyometric training group [8] maintained jumping ability. This suggests that the PLYO intervention had a positive effect in maintaining muscular function and lower body power.

In this population, adaptations made through PLYO training did not transfer to improved distance running performance. Unlike other studies that have shown improved time trial performance in $3 \mathrm{k}$ to $5 \mathrm{k}$ distances and/or improved RE $[4,5,7,8,10,11]$, this study found no difference in $2 \mathrm{MI}$ performance or $\mathrm{RE}$ between the groups. The lack of an effect on distance running performance may be due to a number of factors. First, and perhaps most importantly, is the change in running performance experienced by the group as a whole due to the demanding nature of the marathon training program. The lack of an effect of training group on distance running performance is not entirely surprising, as any adaptation to a 15-20 minute weekly session would be expected to be small in contrast with the relatively high volume running training performed by both groups. Indeed, the effect of time on the distance running performance variables was significant $(P \leq 0.001)$ for the whole population. None of the other studies cited above implemented increases in running training volume concurrent with the plyometric intervention. While the 6-week run-in period in this study represented an attempt to reduce the magnitude of changes due to the running training, the marathon training program required long runs of up to 20 miles, whereas the run-in period had no runs longer than 8 miles. Another study that found no effect of PLYO on RE, 3200-m run or $\mathrm{VO}_{2 \mathrm{MAX}}$ involved high school cross country runners during their summer training [23]. Miles per week at the initiation of the study were not reported, but it was stated that the average participant had run in less than five of the previous six weeks, and then averaged between 25-30 miles per week during the study. Both the experimental and control group improved RE and 3200-m performance, but there was no effect of training group. The use of a population that is more highly trained at baseline, and/or more experienced, may facilitate a clearer picture of the 
effect of PLYO training on distance running performance variables in marathon runners.

The trend toward lower RER and higher $\mathrm{VO}_{2 \mathrm{MAX}}$ in the PLYO group warrants further investigation. While $\mathrm{VO}_{2 \mathrm{MAX}}$ has not been shown to improve from PLYO training in runners $[4,5,10,11,23]$, it may be possible, particularly in a less experienced population that is unaccustomed to higher intensity training. While one of the above studies did use average runners [11], many of the exercises included in the program were either intermittent or sub-maximal (thus arguably outside of the standard definition of plyometric), and no explosive speed training was included. A recent study found an improvement in $\mathrm{VO}_{2 \mathrm{MAX}}$ after 6 weeks of plyometric training, with a larger improvement (though not significantly greater) than the control group [8]. Studies on untrained subjects have shown increases in $\mathrm{VO}_{2 \mathrm{MAX}}$ with high intensity muscular strengthening exercise $[27,28]$. Short, high intensity interval training has been shown to elicit favorable metabolic adaptations such as increased fat utilization [29, 30]. The trend toward lower RER, i.e. increased fat metabolism, may be reflective of similar adaptations. The lower RER values in the PLYO group may also indicate a reduction in energy cost of running, as energy equivalent of oxygen increases as an individual shifts from metabolizing fat to carbohydrate [31]. Because of this, some have argued that RE should be assessed using an energy cost, rather the traditional oxygen cost model [32].

The post-hoc analysis showing a significant interaction between marathon finish time, training group, and performance level should be interpreted with caution. However, it does suggest that a more pointed study of the effects of plyometric and explosive speed training on competitive marathoners is warranted. It is possible that a baseline level of distance running ability is needed in order to be able to transfer the benefits plyometric training to marathon performance. Less experienced individuals may benefit more from a basic strengthening routine such as the core program that served as the control group in this study. Separating the explosive speed and plyometric components of training may also elucidate the effects of these types of training on different sub-groups of marathon runners. In addition, an examination of the biomechanical and cellular mechanisms associated with changes in performance parameters in marathon runners could shed light on the best practices of implementing and monitoring high intensity forms of training in marathon runners.

\section{Conclusion}

The results of this study suggest that plyometric and explosive speed training can effectively be implemented to improve speed and maintain jumping ability in a population of recreational runners concurrently engaged in marathon training. Increased frequency or volume of jump training may be necessary to induce improvements in jumping ability, but that may not be feasible or productive in this population. Unlike some other studies on plyometric training and distance runners, no improvement in distance running performance was observed. This may be due to the magnitude of physiological stress and changes from marathon training alone. The trends toward improved $\mathrm{VO}_{2 \mathrm{MAX}}$ and lower RER in the PLYO group suggest potential performance benefits from plyometric training for marathon runners. The interaction between performance level and training group suggest that plyometric training may have a positive effect on marathon performance in the faster runners, but a negative effect on slower runners. It is possible that a more individualized program, with a lower load on some subjects and a more demanding load on others, could produce stronger effects, but any increased load must be balanced with the potential negative effects of additional physiological stress.

\section{Acknowledgements}

The authors would like to thank the participants in this study, as well as research assistants Jarred 
Sampson and Alena Brooks. We would also like to thank all of the students from the Human and Sport Performance Laboratory for their assistance in this project.

\section{References}

[1] Sáez de Villarreal, E., Requena, B., and Cronin, J. 2012. "The Effects of Plyometric Training on Sprint Performance: A Meta-Analysis." J. Strength Cond. Res. 26 (2): $575-84$.

[2] Markovic, G., and Mikulic, P. 2010. "Neuro-musculoskeletal and Performance Adaptations to Lower-extremity Plyometric Training." Sports Med. 40 (10): 859-95.

[3] Ross, A., Leveritt, M., and Riek, S. 2001. "Neural Influences on Sprint Running." Sports Med. 31 (6): 409-25.

[4] Paavolainen, L. M., Hakkinen, K., Hamalainen, I., Nummela, A. T., and Rusko, H. K. 1999. "Explosive-strength Training Improves 5-km Running Time by Improving Running Economy and Muscle Power." J. Appl. Physiol. 86 (5): 1527-33.

[5] Spurrs, R. W., Murphy, A. J., and Watsford, M. L. 2003. "The Effect of Plyometric Training on Distance Running Performance.” Eur. J. Appl. Physiol. 89 (1): 1-7.

[6] Yamamoto, L. M., Lopez, R. M., Klau, J. F., Casa, D. J., Kraemer, W. J., and Maresh, C. M. 2008. "The Effects of Resistance Training on Endurance Distance Running Performance among Highly Trained Runners: A Systematic Review." J. Strength Cond. Res. 22 (6): 2036.

[7] Ramirez-Campillo, R., Alvarez, C., Henriquez-Olguin, C., Baez, E. B., Martinez, C., Andrade, D. C., and Izquierdo, M. 2014. "Effects of Plyometric Training on Endurance and Explosive Strength Performance in Competitive Middle- and Long-distance Runners." J. Strength Cond. Res. 28 (1): 97-104.

[8] Pellegrino, C., Ruby, L., and Dumke, L. 2016. "Effect of Plyometrics on the Energy Cost of Running and MHC and Titin Isoforms." Med. Sci. Sports Exerc. 48 (1): 49-56.

[9] Barnes, K., and Kilding, A. 2015. "Strategies to Improve Running Economy." Sports Med. 45 (1): 37-56.

[10] Saunders, P. U., Telford, R. D., Pyne, D. B., Peltola, E. M., Cunningham, R. B., Gore, C. J., and Hawley, J. A. 2006. "Short-term Plyometric Training Improves Running Economy in Highly Trained Middle and Long Distance Runners." J. Strength Cond. Res. 20 (4): 947.

[11] Turner, A. M., Owings, M., and Schwane, J. A. 2003. "Improvement in Running Economy after 6 Weeks of Plyometric Training.” J. Strength Cond. Res. 17 (1): 60-7.

[12] Ross, A., and Leveritt, M. 2001. "Long-term Metabolic and Skeletal Muscle Adaptations to Short-sprint Training." Sports Med. 31 (15): 1063-82.

[13] Ronnestad, B. R., and Mujika, I. 2014. "Optimizing Strength Training for Running and Cycling Endurance Performance: A Review." Scand J. Med. Sci. Sports 24 (4): 603-12.

[14] Luden, N., Hayes, E., Minchev, K., Louis, E., Raue, U., Conley, T., and Trappe, S. 2012. "Skeletal Muscle Plasticity with Marathon Training in Novice Runners." Scand. J Med. Sci. Sports 22 (5): 662-70.

[15] Trappe, S., Harber, M., Creer, A., Gallagher, P., and Whitsett, D. 2006. "Single Muscle Fiber Adaptations with Marathon Training.” J. Appl. Physiol. 101 (3): 721-7.

[16] Derman, W., Schwellnus, M. P., Lambert, M. I., Emms, M., Sinclair-Smith, C., Kirby, P., and Noakes, T. D. 1997. "The 'Worn-out Athlete': A Clinical Approach to Chronic Fatigue in Athletes." J. Sports Sci. 15 (3): 341-51.

[17] Grobler, L. A., Collins, M., Lambert, M. I., Sinclair-Smith, C., Derman, W., St Clair Gibson, A., and Noakes, T. D. 2004. "Skeletal Muscle Pathology in Endurance Athletes with Acquired Training Intolerance." Br. J. Sports Med. 38 (6): 697-703.

[18] Koopman, R., and van Loon, L. J. 2009. “Aging, Exercise, and Muscle Protein Metabolism.” J. Appl. Physiol. 106 (6): 2040.

[19] Stanton, R., Reaburn, P. R., and Humphries, B. 2004. "The Effect of Short-term Swiss Ball Training on Core Stability and Running Economy." J. Strength Cond. Res. 18 (3): 522-8.

[20] Arrese, A. L., Izquierdo, D. M., and Serveto Galindo, J. R. 2005. "Physiological Measures Associated with Marathon Running Performance in High-level Male and Female Homogeneous Groups." Int. J. Sports Med. 27 (4): 289-95.

[21] McKelvie, S. J., Valliant, P. M., and Asu, M. E. 1985. "Physical Training and Personality Factors as Predictors of Marathon Time and Training Injury." Percept Mot. Skills 60 (2): 551-66.

[22] Ebben, W. P., Feldmann, C. R., VanderZanden, T. L., Fauth, M. L., and Petushek, E. J. 2010. "Periodized Plyometric Training Is Effective for Women, and Performance Is not Influenced by the Length of post-Training Recovery." J. Strength Cond. Res. 24 (1): $1-7$.

[23] Lathrop, M. C., Womack, C. J., and Paton, C. 2001. "Biomechanical and Physiological Effects of Plyometric Training on Adolescent Cross-country Runners." Int. J. Appl. Sport Sci. 13 (2): 12-26.

[24] Daniels, J., and Gilbert, J. 1979. Oxygen Power: Performance Tables for Distance Runners. J. Daniels, J. Gilbert .

[25] Sàez de Villarreal, E., Kellis, E., Kraemer, W. J., and 
Izquierdo, M. 2009. "Determining Variables of Plyometric Training for Improving Vertical Jump Height Performance: A Meta-analysis.” J. Strength Cond. Res. 23 (2): 495.

[26] Losnegard, T., Mikkelsen, K., Rønnestad, B., Hallén, J., Rud, B., and Raastad, T. 2011. "The Effect of Heavy Strength Training on Muscle Mass and Physical Performance in Elite cross Country Skiers." Scand J. Med. Sci. Sports. 21 (3): 389.

[27] Stone, M. H., Wilson, G. D., Blessing, D., and Rozenek, R. 1983. "Cardiovascular Responses to Short-term Olympic Style Weight-training in Young Men.” Can. J. Appl. Sport. Sci 8 (3): 134.

[28] McCarthy, J. P., Agre, J. C., Graf, B. K., Pozniak, M. A., and Vailas, A. C. 1995. "Compatibility of Adaptive Responses with Combining Strength and Endurance Training.” Med. Sci. Sports Exerc. 27 (3): 429.
[29] Gibala, M. J. 2006. "Short-term Sprint Interval versus Traditional Endurance Training: Similar Initial Adaptations in Human Skeletal Muscle and Exercise Performance." J. Physiol. 575 (3): 901-11.

[30] Burgomaster, K. A., Howarth, K. R., Phillips, S. M., Rakobowchuk, M., Macdonald, M. J., McGee, S. L., and Gibala, M. J. 2008. "Similar Metabolic Adaptations during Exercise after Low Volume Sprint Interval and Traditional Endurance Training in Humans." J. Physiol. 586 (1): 151-60.

[31] Peronnet, F., and Massicotte, D. 1991. "Table of Nonprotein Respiratory Quotient: An Update." Can. J. Appl. Sport Sci. 16 (1): 23-9.

[32] Fletcher, J. R., Esau, S. P., and MacIntosh, B. R. 2009. "Economy of Running: Beyond the Measurement of Oxygen Uptake.” J. Appl. Physiol. 107 (6): 1918-22. 